"This is the peer reviewed version of the following article: [Benchmarked Risk Minimization], which has been published in final form at [10.1111/mafi.12065].

This article may be used for non-commercial purposes in accordance with Wiley Terms and Conditions for Self-Archiving." 


\title{
Benchmarked Risk Minimization
}

\author{
$\mathrm{Ke} \mathrm{Du}^{1}$ and Eckhard Platen ${ }^{2}$
}

December 13, 2013

\begin{abstract}
This paper discusses the problem of hedging not perfectly replicable contingent claims using the numéraire portfolio. The proposed concept of benchmarked risk minimization leads beyond the classical no-arbitrage paradigm. It provides in incomplete markets a generalization of the pricing under classical risk minimization, pioneered by Föllmer, Sondermann and Schweizer. The latter relies on a quadratic criterion, requests square integrability of claims and gains processes, and relies on the existence of an equivalent risk neutral probability measure. Benchmarked risk minimization avoids these restrictive assumptions and provides symmetry with respect to all primary securities. It employs the real world probability measure and the numéraire portfolio to identify the minimal possible price for a contingent claim. Furthermore, the resulting benchmarked (i.e. numéraire portfolio denominated) profit and loss is only driven by uncertainty that is orthogonal to benchmarked traded uncertainty, and forms a local martingale that starts at zero. Consequently, sufficiently different benchmarked profits and losses, when pooled, become asymptotically negligible through diversification. This property makes benchmarked risk minimization the least expensive method for pricing and hedging diversified pools of not fully replicable benchmarked contingent claims. Additionally, when hedging it incorporates evolving information about non-hedgeable uncertainty, which is ignored under classical risk minimization.
\end{abstract}

JEL Classification: G10, G13

1991 Mathematics Subject Classification: primary 65C20; secondary 60H10. Key words and phrases: risk minimization, incomplete market, pricing, hedging, numéraire portfolio, benchmark approach.

\footnotetext{
${ }^{1}$ Southwestern University of Finance and Economics, Institute of Financial Studies, No. 55, Guanghuacun Street, Chengdu, Sichuan, P.R. China

${ }^{2}$ University of Technology, Sydney, Finance Discipline Group and School of Mathematical Sciences, PO Box 123, Broadway, NSW, 2007, Australia
} 


\section{Introduction}

There has been a growing literature that pays attention to models that exhibit "anomalies" that cannot be accommodated by classical no-arbitrage theory. For instance, an equivalent risk neutral probability measure may not exist in some of these models, see e.g. Delbaen and Schachermayer (1995), Loewenstein and Willard (2000), Fernholz (2002), Platen (2002, 2006), Fernholz et al. (2005), Platen and Heath (2006), Jarrow et al. (2010), Karatzas and Kardaras (2007), Heston et al. (2007), Christensen and Larsen (2007), Fernholz and Karatzas (2009), Galesso and Runggaldier (2010), Fernholz and Karatzas (2010), Biagini (2011) and Davis and Lleo (2011). Heath and Platen (2002) and Fernholz et al. (2005) demonstrated that pricing and hedging is still possible outside the classical no-arbitrage framework. The results in Fernholz (2002), Platen (2002), Fernholz et al. (2005) and Platen and Heath (2006) indicate that for realistic long term modelling one has, most likely, to abandon the classical no-arbitrage paradigm.

A general framework for pricing and hedging in incomplete markets, which can handle also models outside the classical no-arbitrage framework, is provided by the benchmark approach, described in Platen (2002, 2006) and Platen and Heath (2006). A similar framework for pricing and hedging in complete markets beyond the classical theory has been suggested in Fernholz et al. (2005). Under the benchmark approach, asset prices are modeled under the real world probability measure and the corresponding numéraire is the numéraire portfolio (NP). This portfolio, which was originally studied by Kelly (1956), maximizes expected log-utility. When the NP is taken as numéraire, pricing can be conveniently performed under the real world probability measure, see Long (1990) and Platen (2002). Under the benchmark approach the existence of an equivalent risk neutral probability measure is not required. The benchmark approach generalizes the classical risk neutral approach. The normalized benchmarked savings account, with the NP as benchmark, is then the Radon-Nikodym derivative of the putative risk neutral measure.

The pricing and hedging of not fully replicable contingent claims has been a challenging task. Strategies that aim to replicate such a contingent claim generate usually a fluctuating profit and loss process. The risk minimization approach of Föllmer and Sondermann (1986), further developed in Föllmer and Schweizer (1989) and Schweizer (1991, 2000), minimizes fluctuations of discounted profit and loss processes by using a quadratic criterion under an assumed risk neutral probability measure. In principle, it introduces an account, which monitors in units of the savings account the adapted inflow and outflow of capital to and from the hedge portfolio. The resulting discounted profit and loss process forms a local martingale under the assumed equivalent risk neutral probability measure, referred to as the minimal equivalent martingale measure, and is under this measure orthogonal to discounted traded wealth. This provides an intuitively appealing methodology for pricing and hedging contingent claims that cannot be perfectly replicated.

Hereafter, we refer to the above approach as the Föllmer-Sondermann-Schweizer 
approach or the classical risk minimization approach. Despite the appealing properties of classical risk minimization, this approach creates some asymmetries among primary securities by using the domestic savings account as numéraire, and it makes the restrictive assumption on the existence of the minimal equivalent martingale measure. The existence of such measure in the case with jumps is not easily established, see Schweizer (2000). Moreover, certain second moments have to exist.

We will see that diversification of hedge errors in a large trading book occurs according to the Law of Large Numbers under the real world probability measure. In Schweizer (2000), see also Biagini et al. (2011), the concept of local risk minimization, introduced in Schweizer (1991), was generalized with a view towards the real world probability measure. However, asymmetries with respect to primary security accounts and second moment conditions still remain present in this generalization.

The current paper proposes the concept of benchmarked risk minimization for pricing and hedging contingent claims which cannot be perfectly replicated in an incomplete semimartingale market. It does not assume the existence of an equivalent risk neutral probability measure or minimal equivalent martingale measure. It generalizes the pricing under classical risk minimization, and allows one to price and hedge in models beyond the risk neutral paradigm. Symmetry with respect to all primary security accounts will be secured, and second moment assumptions will be avoided.

The resulting pricing rule is that of real world pricing, with the NP as numéraire and the real world probability measure as pricing measure. Under benchmarked risk minimization the minimal possible price for a contingent claim is obtained. When a minimal equivalent martingale measure exists, then real world pricing coincides with the pricing under classical risk minimization. The remaining benchmarked (numéraire portfolio denominated) profit and loss process forms a local martingale. It starts at zero and is orthogonal to the benchmarked primary security accounts, in the sense that the products with these are local martingales.

A benchmarked risk minimizing hedging strategy minimizes the fluctuations of the benchmarked nonhedgeable part of a benchmarked contingent claim. Moreover, during hedging it takes evolving information about the nonhedgeable part of the claim into account, whereas classical risk minimization ignores such information. This is an important property of BRM strategies. The total benchmarked profit and loss of a trading book becomes asymptotically negligible when holding an increasing number of sufficiently different benchmarked profit and loss processes.

The paper is organized as follows: Section 2 presents a general semimartingale market. In Section 3 the real world pricing formula is derived. Section 4 considers benchmarked profit and loss processes. The concept of benchmarked risk minimization is introduced in Section 5. Section 6 links martingale representations and benchmarked risk minimization. Section 7 derives the respective hedging strategy. In Section 8 a quadratic criterion is illustrated with its link to benchmarked risk minimization. Section 9 discusses real world and risk neutral pricing. Finally, Section 10 emphasises differences in hedging between benchmarked risk minimization and classical risk minimization. 


\section{Financial Market}

In this paper we consider a semimartingale financial market in continuous time. Assume a filtered probability space $(\Omega, \mathcal{F}, \underline{\mathcal{F}}, P)$ that satisfies the usual conditions, as described in Protter (2005). Here, the sigma field $\mathcal{F}_{t}$ models the information available at time $t \in[0, \infty)$. The filtration $\underline{\mathcal{F}}=\left(\mathcal{F}_{t}\right)_{t \in[0, \infty)}$ describes the evolution of market information over time. $P$ denotes the real world probability measure. In this market we consider $d \in\{1,2, \ldots\}$ adapted, non-negative assets, which we call primary security accounts, where all interests and dividends are reinvested. We assume that a numéraire portfolio (NP) exists such that every nonnegative primary security account process $\hat{S}^{j}=\left\{\hat{S}_{t}^{j}, t \in[0, \infty)\right\}, j \in\{1, \ldots, d\}$, when expressed in units of the NP, forms a right-continuous, integrable $(\underline{\mathcal{F}}, P)$-local martingale and, thus, an $(\underline{\mathcal{F}}, P)$-supermartingale, see e.g. Platen (2002), Platen and Heath (2006). Karatzas and Kardaras (2007) provide general conditions for the existence of a NP. Hereafter, we refer to prices, when denominated in units of the NP, as benchmarked prices. Denote by $[\hat{\mathbf{S}}]=\left\{[\hat{\mathbf{S}}]_{t}=\left(\left[\hat{S}^{i}, \hat{S}^{j}\right]_{t}\right)_{i, j=1}^{d}, t \in[0, \infty)\right\}$ the matrix valued optional covariation process of the vector process of benchmarked primary security accounts $\hat{\mathbf{S}}=\left\{\hat{\mathbf{S}}_{t}=\left(\hat{S}_{t}^{1}, \ldots, \hat{S}_{t}^{d}\right)^{\top}, t \in[0, \infty)\right\}$.

We denote by $S_{t}^{i, i}$ the $i$ th primary security account value at time $t \in[0, \infty)$, when denominated in units of the $i$ th security itself, $i \in\{1, \ldots, d\}$. In the case of the $i$ th currency denomination, $S_{t}^{i, i}$ denotes the savings account in units of this currency. In the case when the $i$ th shares are used for denomination, $S_{t}^{i, i}$ denotes the respective share savings account in units of those shares. Then the NP value $S_{t}^{i, \delta *}$, when denominated at time $t$ in units of the $i$ th security, can be expressed by the ratio

$$
S_{t}^{i, \delta *}=\frac{S_{t}^{i, i}}{\hat{S}_{t}^{i}},
$$

for $t \in[0, \infty), i \in\{1, \ldots, d\}$. Consequently, the $j$ th primary security account $S_{t}^{i, j}$, when denominated at time $t$ in units of the $i$ th security, can be obtained as the product

$$
S_{t}^{i, j}=\hat{S}_{t}^{j} S_{t}^{i, \delta *}
$$

for $i, j \in\{1, \ldots, d\}, t \in[0, \infty)$.

The market participants can combine primary security accounts to form portfolios. Denote by $\boldsymbol{\delta}=\left\{\boldsymbol{\delta}_{t}=\left(\delta_{t}^{1}, \ldots, \delta_{t}^{d}\right)^{\top}, t \in[0, \infty)\right\}$ the strategy, where $\delta_{t}^{j}, j \in\{1, \ldots, d\}$, represents the number of units of the $j$ th primary security account that are held at time $t$ in a corresponding portfolio. When denominated in units of the NP, this portfolio is denoted by the benchmarked portfolio process $\hat{S}^{\delta}=\left\{\hat{S}_{t}^{\delta}, t \in[0, \infty)\right\}$, where

$$
\hat{S}_{t}^{\delta}=\boldsymbol{\delta}_{t}^{\top} \hat{\mathbf{S}}_{t}
$$

for $t \in[0, \infty)$.

If changes in the value of a portfolio are only due to changes in the values of the primary security accounts, then no extra funds flow in or out of the portfolio, and the 
corresponding portfolio and strategy are called self-financing. This property can be expressed by the equation

$$
\hat{S}_{t}^{\delta}=\hat{S}_{0}^{\delta}+\int_{0}^{t} \boldsymbol{\delta}_{s}^{\top} d \hat{\mathbf{S}}_{s}
$$

for all $t \in[0, \infty)$, where the stochastic integral in (2.4) is a vector Itô integral. Since each benchmarked primary security account process $\hat{S}^{j}, j \in\{1, \ldots, d\}$, is a local martingale, the benchmarked self-financing portfolio $\hat{S}^{\delta}$ is also a local martingale. A benchmarked non-negative, self-financing portfolio is, therefore, a supermartingale by Fatou's Lemma. This confirms the defining property of the NP of being the strictly positive portfolio that when used as benchmark makes all benchmarked nonnegative portfolios supermartingales, see Long (1990), Becherer (2001) and Karatzas and Kardaras (2007).

Dynamic trading strategies that may not be self-financing are crucial for risk management. Obviously, not all strategies can be allowed. It is sensible to focus in the following on strategies that are consistent with the fact that the NP is the "best" performing portfolio in the sense that they yield benchmarked nonnegative price processes that are supermartingales. Now, let us introduce a class of admissible strategies that can form non-self-financing portfolios.

Definition 2.1. A dynamic trading strategy $\boldsymbol{v}$, initiated at time $t=0$, is an $\mathbf{R}^{d+1}$ valued stochastic process $\boldsymbol{v}=\left\{\boldsymbol{v}_{t}=\left(\eta_{t}, \vartheta_{t}^{1}, \ldots, \vartheta_{t}^{d}\right)^{\top}, t \in[0, \infty)\right\}$, where $\boldsymbol{\vartheta}=\left\{\boldsymbol{\vartheta}_{t}=\right.$ $\left.\left(\vartheta_{t}^{1}, \ldots, \vartheta_{t}^{d}\right)^{\top}, t \in[0, \infty)\right\}$ describes the number of units invested in the benchmarked primary security accounts to form at time $t$ the self-financing part $\boldsymbol{\vartheta}_{t}^{\top} \hat{\mathbf{S}}_{t}$ of the associated portfolio. The right continuous benchmarked price process $\hat{V}^{\boldsymbol{v}}=\left\{\hat{V}_{t}^{\boldsymbol{v}}, t \in[0, \infty)\right\}$ of the associated portfolio is a supermartingale and given by the sum

$$
\hat{V}_{t}^{\boldsymbol{v}}=\boldsymbol{\vartheta}_{t}^{\top} \hat{\mathbf{S}}_{t}+\eta_{t}
$$

at time $t \in[0, \infty)$. Here $\boldsymbol{\vartheta}$ is assumed to be an $\mathbf{R}^{d}$-valued, predictable process satisfying

$$
\int_{0}^{t} \boldsymbol{\vartheta}_{u}^{\top} d[\hat{\mathbf{S}}]_{u} \boldsymbol{\vartheta}_{u}<\infty
$$

for all $t \in[0, \infty)$. The adapted, scalar process $\eta=\left\{\eta_{t}, t \in[0, \infty)\right\}$, starting with initial value $\eta_{0}=0$, monitors the benchmarked non-self-financing part of the benchmarked price process $\hat{V}^{\boldsymbol{v}}$, so that

$$
\hat{V}_{t}^{\boldsymbol{v}}=\hat{V}_{0}^{\boldsymbol{v}}+\int_{0}^{t} \boldsymbol{\vartheta}_{s}^{\top} d \hat{\mathbf{S}}_{s}+\eta_{t}
$$

for $t \in[0, \infty)$, where the stochastic integral in (2.7) is a vector Itô integral.

With the above notion of a dynamic trading strategy one can model a wide range of benchmarked price processes. Later we will restrict the above class of admissible 
dynamic trading strategies when introducing the concept of benchmarked risk minimization.

We emphasize that a dynamic trading strategy generates via its self-financing part $\boldsymbol{\vartheta}_{t}^{\top} \hat{\mathbf{S}}_{t}$ the benchmarked gains from trade

$$
\int_{0}^{t} \boldsymbol{\vartheta}_{s}^{\top} d \hat{\mathbf{S}}_{s}=\boldsymbol{\vartheta}_{t}^{\top} \hat{\mathbf{S}}_{t}-\hat{V}_{0}^{\boldsymbol{v}}
$$

It does this in a manner that does not require outside funds and also does not generate extra funds. In general, capital has to be added or removed from a portfolio so that its benchmarked value matches the evolution of a given benchmarked price process $\hat{V}^{\boldsymbol{v}}$. We will see that for risk management purposes it is enough to monitor, here in units of the NP, the cumulative amount $\eta_{t}$, which has to be added or removed from the portfolio to match a desired price $\hat{V}_{t}^{\boldsymbol{v}}$ at time $t \in[0, \infty)$.

The predictability of the integrand in the benchmarked gains from trade (2.8) expresses the real informational constraint that the allocation expressed in $\boldsymbol{\vartheta}$ is not allowed to anticipate the movements of $\hat{\mathbf{S}}$. This predictability is also theoretically needed for the integrand in 2.8 to yield a proper vector Itô integral with respect to the vector of benchmarked primary security account processes. The monitoring process $\eta$ in (2.7) needs only to be adapted, which is less restrictive than the predictability required for the components of the process $\boldsymbol{\vartheta}$.

Via the process $\eta$ the investor monitors in units of the NP the cumulative "virtual" capital inflow and outflow from the portfolio. In previous work by Föllmer and Sondermann (1986) and Schweizer (2000), a similar adapted process was employed for describing the holdings in their numéraire, the domestic savings account. This choice of numéraire creates some asymmetry in the requested measurability properties among all primary security accounts. The dynamic trading strategy, introduced in Definition 2.1. employs the NP as numéraire and monitors the inflow and outflow of extra capital in units of the NP. This choice of numéraire brings all primary security accounts into comparable positions, including the domestic savings account.

Note, if there is no inflow or outflow of capital in a dynamic trading strategy, then one deals with a self-financing portfolio, as described in (2.4). More generally, when allowing extra capital inflows and outflows, one obtains directly from Definition 2.1 the following result:

Corollary 2.2. For a dynamic trading strategy $\boldsymbol{v}=\left\{\boldsymbol{v}_{t}=\left(\eta_{t}, \vartheta_{t}^{1}, \ldots, \vartheta_{t}^{d}\right)^{\top}, t \in\right.$ $[0, \infty)\}$, as introduced in Definition 2.1, the benchmarked portfolio is given by

$$
\hat{V}_{t}^{\boldsymbol{v}}=\hat{S}_{t}^{\delta}=\boldsymbol{\delta}_{t}^{\top} \hat{\mathbf{S}}_{t}
$$

with

$$
\boldsymbol{\delta}_{t}=\boldsymbol{\vartheta}_{t}+\eta_{t} \boldsymbol{\delta}_{*}(t)
$$

Here $\boldsymbol{\delta}_{*}(t)=\left(\delta_{*}^{1}(t) \ldots \delta_{*}^{d}(t)\right)^{\top}$, denotes the vector of numbers of units of the respective primary security accounts held in the NP at time $t$. We have for the benchmarked NP 
the trivial equality

$$
\hat{S}_{t}^{\delta_{*}}=\boldsymbol{\delta}_{*}(t) \hat{\mathbf{S}}_{t}=1
$$

for $t \in[0, \infty)$.

We remark that $\boldsymbol{\delta}$ in 2.10 is, in general, not predictable since $\eta$ needs only to be adapted. Furthermore, we note that a dynamic trading strategy has still some ambiguity in what constitutes for a given price process its self-financing part and what its monitoring part. This ambiguity will be removed in Section 5 when introducing the concept of benchmarked risk minimization.

\section{Real World Pricing}

The main aim of hedging is risk minimization for the delivery of a targeted payoff via some dynamic trading strategy. Fix a bounded stopping time $T>0$, and let $\mathcal{L}^{1}\left(\mathcal{F}_{T}\right)$ denote the set of integrable $\mathcal{F}_{T}$-measurable random variables.

Definition 3.1. For a bounded stopping time $T \in(0, \infty)$ a nonnegative payoff $\hat{H}_{T} \in$ $\mathcal{L}^{1}\left(\mathcal{F}_{T}\right)$, denominated in units of the $N P$, is called a benchmarked contingent claim.

Since one can decompose a general payoff into its nonnegative and negative part, there is no real restriction imposed when considering in Definition 3.1 nonnegative payoffs.

Definition 3.2. We say, a dynamic trading strategy $\boldsymbol{v}=\left\{\boldsymbol{v}_{t}=\left(\eta_{t}, \vartheta_{t}^{1}, \ldots, \vartheta_{t}^{d}\right)^{\top}, t \in\right.$ $[0, \infty)\}$ delivers the benchmarked contingent claim $\hat{H}_{T}$ if

$$
\hat{V}_{T}^{\boldsymbol{v}}=\hat{H}_{T}
$$

P-a.s. A benchmarked contingent claim is called replicable if there exists a selffinancing dynamic trading strategy $\boldsymbol{v}$ with $\eta_{t}=0$ P-a.s for all $t \in[0, T]$, which delivers the claim.

There may exist several self-financing strategies that deliver a given benchmarked contingent claim. Examples can be found in Platen (2002), Fernholz et al. (2005) and Platen and Heath (2006). The defining property of the NP ensures that all non-negative, self-financing portfolios, when benchmarked, are supermartingales. We show in Appendix A that in a set of nonnegative supermartingales, which replicate a given benchmarked contingent claim, the minimal nonnegative supermartingale is the martingale. This crucial fact yields the following result:

Proposition 3.3. If for a given benchmarked contingent claim $\hat{H}_{T}$ a self-financing benchmarked portfolio $\hat{S}^{\delta_{H_{T}}}$ exists, satisfying the real world pricing formula

$$
\hat{S}_{t}^{\delta_{\hat{H}_{T}}}=E\left(\hat{H}_{T} \mid \mathcal{F}_{t}\right)
$$

for all $t \in[0, T] P$-a.s., then this portfolio provides the least expensive hedge for $\hat{H}_{T}$. 
Proof. This result follows directly from the application of Lemma 11.1 in Appendix A.

Note that equation (3.2) provides the minimal possible price for a fully replicable claim. In general, contingent claims may be not fully replicable. We will show in Section 5 that the above real world pricing formula $(3.2)$ also makes perfect sense for non-replicable claims.

\section{Benchmarked Profit and Loss}

Risk can be reduced by hedging and diversification. Hedging a non-replicable contingent claim usually results in a hedge error. The current paper aims to identify the least expensive way of delivering contingent claims through hedging, while minimizing the fluctuations of the benchmarked hedge error. The following notion will allow us to keep track of benchmarked hedge errors.

Definition 4.1. For a dynamic trading strategy $\boldsymbol{v}=\left\{\boldsymbol{v}_{t}=\left(\eta_{t}, \vartheta_{t}^{1}, \ldots, \vartheta_{t}^{d}\right)^{\top}, t \in\right.$ $[0, \infty)\}$, with benchmarked price $\hat{V}_{t}^{\boldsymbol{v}}$ at time $t \in[0, \infty)$, the benchmarked profit and loss (P\&L) process $\hat{C}^{\boldsymbol{v}}=\left\{\hat{C}_{t}^{\boldsymbol{v}}, t \in[0, \infty)\right\}$ is defined as

$$
\hat{C}_{t}^{\boldsymbol{v}}=\hat{V}_{t}^{\boldsymbol{v}}-\int_{0}^{t} \boldsymbol{\vartheta}_{u}^{\top} d \hat{\mathbf{S}}_{u}-\hat{V}_{0}^{\boldsymbol{v}}
$$

for $t \in[0, \infty)$.

One obtains directly from Definition 4.1 with Definition 2.1 the following statement:

Corollary 4.2. For a dynamic trading strategy $\boldsymbol{v}=\left\{\boldsymbol{v}_{t}=\left(\eta_{t}, \vartheta_{t}^{1}, \ldots, \vartheta_{t}^{d}\right)^{\top}, t \in[0, \infty)\right\}$ the corresponding benchmarked $P \& L$ process $\hat{C}^{\boldsymbol{v}}=\left\{\hat{C}_{t}^{\boldsymbol{v}}, t \in[0, \infty)\right\}$ coincides with the adapted process $\eta=\left\{\eta_{t}, t \in[0, \infty)\right\}$ that monitors the cumulative inflow and outflow of extra capital.

Intuitively, the adapted process $\eta$ can be interpreted as benchmarked hedge error. For convenience in the current paper, for a given dynamic trading strategy $\boldsymbol{v}$ the hedging and, thus, the benchmarked P\&L process $\hat{C}^{\boldsymbol{v}}$ are assumed to start at the initial time $t=0$. Therefore, the benchmarked P\&L has initial value $\hat{C}_{0}^{\boldsymbol{v}}=\eta_{0}=0$ and monitors at time $t$ with $\hat{C}_{t}^{\boldsymbol{v}}=\eta_{t}$ the adapted accumulated benchmarked capital that flew in or out of the respective portfolio that matches the benchmarked price process $\hat{V}^{\boldsymbol{v}}$ until this time. In other words, $\hat{C}_{t}^{\boldsymbol{v}}$ represents the benchmarked external costs incurred by the dynamic trading strategy $\boldsymbol{v}$ over the time period $[0, t]$ after the hedge was set up at the initial time zero. If one has to deliver a general claim, one faces a fluctuating benchmarked P\&L process and, thus, an intrinsic risk that needs to be controlled. For implementing systematically such a control one can introduce a criterion to obtain a desirable behavior of the benchmarked P\&L process. The question is, what criterion would be most appropriate from a risk management point of view?

To get an idea about what criterion to choose, we look at the broader picture and prove the following motivating result: 
Proposition 4.3. Consider benchmarked contingent claims $\hat{H}_{T, l}, l \in\{1,2, \ldots\}$, with respective price processes $\hat{V}^{\boldsymbol{v}_{l}}, l \in\{1,2, \ldots\}$, and benchmarked P\&L processes $\hat{C}^{\boldsymbol{v}_{l}}$, $l \in\{1,2, \ldots\}$, where the latter form independent square integrable martingales with $E\left(\left(\frac{\hat{C}_{t} \hat{\boldsymbol{v}}_{l}}{\hat{V}_{0} \boldsymbol{v}_{l}}\right)^{2}\right) \leq K_{t}<\infty$ for $l \in\{1,2, \ldots\}$ and $t \in[0, T], T \in[0, \infty)$. Assume, for simplicity, that at the initial time the considered well-diversified trading book of a financial institution holds equal fractions of the managed initial benchmarked wealth $\hat{U}_{0}$ in the first $m$ of the contingent claims, such that its total benchmarked wealth at time $t \in[0, T]$ accounts to $\hat{U}_{t}=\frac{\hat{U}_{0}}{m} \sum_{l=1}^{m} \frac{\hat{V}_{t}}{\hat{V}_{0}}$. The total benchmarked $P \& L \hat{R}_{m}(t)$ of the trading book has then at time $t \in[0, T]$ the value

$$
\hat{R}_{m}(t)=\frac{\hat{U}_{0}}{m} \sum_{l=1}^{m} \frac{\hat{C}_{t}^{\boldsymbol{v}_{l}}}{\hat{V}_{0}^{\boldsymbol{v}_{l}}}
$$

and it follows for increasing number $m$ of claims in the trading book that the total benchmarked $P \& L$ vanishes almost surely, that is,

$$
\lim _{m \rightarrow \infty} \hat{R}_{m}(t)=0
$$

P-a.s.

The proof of this rather illuminating fact is given in Appendix B. It shows that the benchmarked P\&L of a trading book with increasing number of claims can be asymptotically removed, which can be interpreted as the process of diversification. The insight that such removal is, in principle, possible is crucial. We emphasise, for the above result to hold it is important that the benchmarked P\&Ls are locally in time close to martingales. We reflect this, by requesting below that benchmarked P\&Ls should be local martingales under the dynamic trading strategies that we will admit. This means, a benchmarked P\&L is locally in the mean self-financing. Mean-self-financing turns out to be an extremely useful notion, which was introduced in Schweizer (1991) when using the savings account as numéraire and employing an assumed risk neutral probability measure as pricing measure. Under the benchmark approach we use the NP as numéraire and the real world probability measure for taking expectations. Hence, the following notion will be employed when introducing in the next section the concept of benchmarked risk minimization:

Definition 4.4. A dynamic trading strategy $\boldsymbol{v}=\left\{\boldsymbol{v}_{t}=\left(\eta_{t}, \vartheta_{t}^{1}, \ldots, \vartheta_{t}^{d}\right)^{\top}, t \in[0, \infty)\right\}$ is called locally real world mean-self-financing if its monitoring process $\eta$ is a local martingale.

This notion maintains symmetry with respect to all primary security accounts, including the domestic savings account. It uses the real world probability measure $P$ and avoids the restrictive assumption on the existence of an equivalent risk neutral probability measure. 


\section{Benchmarked Risk Minimization}

It is not immediately obvious how to price and hedge a general contingent claim in an incomplete market, even when taking into account the observations made above. For complete markets the pricing and hedging of contingent claims can be performed in a straightforward manner also for models where no equivalent risk neutral probability measure exists. This was observed in Fernholz et al. (2005), and under the benchmark approach demonstrated in Heath and Platen (2002), Platen (2002) and Platen and Heath (2006).

Conceptually, there exist many ways to hedge a non-replicable claim, and a wide range of literature has emerged. The pricing in incomplete markets and some pricing of non-replicable contingent claims have been discussed, for instance, in Sections 11.4 and 11.5 in Platen and Heath (2006). Intuitively appealing and practically useful is the already mentioned concept of classical risk minimization for which an excellent survey is given in Schweizer (2000).

Under classical risk minimization along the lines of Föllmer-Sondermann-Schweizer, the hedging is implemented via a savings account discounted portfolio under an assumed equivalent risk neutral probability measure. The fluctuations of the discounted P\&L processes are measured and minimized via a quadratic criterion, where a "good" strategy turns out to be mean-self-financing under the assumed risk neutral probability measure, see Schweizer (2000).

Most importantly, the Föllmer-Sondermann-Schweizer approach links the optimization problem of risk minimization to the well-known Kunita-Watanabe decomposition, see Schweizer (2000). This crucial decomposition became known as FöllmerSchweizer decomposition in the context of pricing and hedging in incomplete markets. The Föllmer-Schweizer decomposition has been extensively studied by several authors, where we refer to Schweizer (2000) for a list of references.

The current paper is of conceptual nature, and proposes a pricing and hedging approach for non-replicable claims in incomplete markets in the spirit of classical risk minimization, but under the real world probability measure with the NP as numéraire. It generalizes the pricing suggested by classical risk minimization. However, the hedging will be, in general, different.

Recall from Definition 2.1 that dynamic trading strategies form benchmarked nonnegative price processes that are consistent with the fact that the NP is the "best" performing portfolio, in the sense that benchmarked price processes form supermartingales. Note also that, at this stage, for a given benchmarked price process a corresponding locally real world mean-self financing dynamic trading strategy remains potentially exposed to some ambiguity concerning what forms its self-financing part and what constitutes its monitoring part, see equation (2.10). This ambiguity will be removed by focusing below on benchmarked P\&Ls with fluctuations that are "orthogonal" to those of the benchmarked primary security accounts under the real world probability measure. This means, intuitively, these fluctuations, when denominated in units of the benchmark, have no chance to be removed via hedging. To formalize this 
idea we introduce the following notion:

Definition 5.1. A dynamic trading strategy $\boldsymbol{v}=\left\{\boldsymbol{v}_{t}=\left(\eta_{t}, \vartheta_{t}^{1}, \ldots, \vartheta_{t}^{d}\right)^{\top}, t \in[0, \infty)\right\}$ has an orthogonal benchmarked $P \& L \eta=\left\{\eta_{t}, t \in[0, \infty)\right\}$ if $\eta$ is orthogonal to the benchmarked primary securities in the sense that $\eta_{t} \hat{\mathbf{S}}_{t}$ forms a vector local martingale.

In some sense, all hedgeable benchmarked uncertainty is removed from an orthogonal benchmarked P\&L. To summarize the so far identified desirable properties of dynamic trading strategies, let us define the following set:

Definition 5.2. For a benchmarked contingent claim $\hat{H}_{T}$, let $\mathcal{V}_{\hat{H}_{T}}$ denote the set of locally real world mean-self-financing dynamic trading strategies, which deliver $\hat{H}_{T}$ with orthogonal benchmarked P\&Ls.

There may exist several dynamic trading strategies in $\mathcal{V}_{\hat{H}_{T}}$ that could deliver the benchmarked contingent claim $\hat{H}_{T}$. To finalize our search for a suitable criterion, we assume that a market participant always prefers more for less. The following definition selects then the most economical price process, which is the least expensive possible price process.

Definition 5.3. A dynamic trading strategy $\widetilde{\boldsymbol{v}}=\left\{\widetilde{\boldsymbol{v}}_{t}=\left(\widetilde{\eta}_{t}^{1}, \widetilde{\vartheta}_{t}^{1}, \ldots, \widetilde{\vartheta}_{t}^{d}\right)^{\top}, t \in[0, T]\right\} \in$ $\mathcal{V}_{\hat{H}_{T}}$, with corresponding benchmarked price process $\hat{V}^{\widetilde{\boldsymbol{v}}}$, is called benchmarked risk minimizing (BRM) if for all dynamic trading strategies $\boldsymbol{v} \in \mathcal{V}_{\hat{H}_{T}}$, with corresponding price process $\hat{V}^{\boldsymbol{v}}$, the price process $\hat{V}^{\tilde{\boldsymbol{v}}}$ is minimal in the sense that

$$
\hat{V}_{t}^{\widetilde{\boldsymbol{v}}} \leq \hat{V}_{t}^{\boldsymbol{v}}
$$

$P$-a.s. for all $t \in[0, T]$.

As required by the inequality (5.1), and similarly as in Section 3, we can exploit the fact that the martingale among the nonnegative supermartingales contained in $\mathcal{V}_{\hat{H}_{T}}$ yields the minimal possible benchmarked price process; see Lemma 11.1 in Appendix A. Therefore, we obtain directly the following result:

Corollary 5.4. For given $\hat{H}_{T} \in \mathcal{L}^{1}\left(\mathcal{F}_{T}\right)$ a BRM dynamic trading strategy $\boldsymbol{v}=\left\{\boldsymbol{v}_{t}=\right.$ $\left.\left(\eta_{t}, \vartheta_{t}^{1}, \ldots, \vartheta_{t}^{d}\right)^{\top}, t \in[0, T]\right\}$ forms with the corresponding benchmarked price process $\hat{V}^{\boldsymbol{v}}$ a martingale, that is, it satisfies the real world pricing formula

$$
\hat{V}_{t}^{\boldsymbol{v}}=E\left(\hat{H}_{T} \mid \mathcal{F}_{t}\right)
$$

$P$-a.s. for $t \in[0, T]$.

This is an intuitively appealing and practically useful conclusion. Obviously, formula (5.2) extends the real world pricing formula (3.2) to the case of not fully replicable benchmarked contingent claims. 
Note that according to the real world pricing formula $(5.2)$, the benchmarked price process is unique and does not depend on the time when the hedge is initiated. However, the benchmarked P\&L process depends on the initiation time of the hedge, see (4.1). Its specification follows from the request that it should be a local martingale that is orthogonal to all benchmarked primary security accounts, see Definitions 5.1 and 5.2 .

Benchmarked risk minimization does not require the existence of an equivalent risk neutral probability measure. It aims for the minimal possible price process. Furthermore, in a trading book with an increasing number of sufficiently different contingent claims it can potentially remove nonhedgeable risk via diversification, as indicated in Proposition 4.3. Moreover, it provides symmetry with respect to all primary security accounts. Finally, restrictive square integrability assumptions are avoided.

Since the proposed concept of benchmarked risk minimization requires only very weak assumptions, it permits the handling of more general financial market models and more general contingent claims than covered under classical risk minimization. Its main requirement is the existence of the NP, which is a very weak assumption, as shown in Karatzas and Kardaras (2007).

\section{Regular Benchmarked Contingent Claims}

To utilize efficiently the above introduced concept of BRM strategies, it will be extremely useful to have access to corresponding martingale representations for benchmarked contingent claims, similar to the Föllmer-Schweizer decomposition in classical risk minimization, see Schweizer (2000). We emphasize, in the current paper we will use martingale representations for benchmarked continent claims under the real world probability measure.

Unfortunately, martingale representations cannot be easily mathematically guaranteed for general semimartingale markets. Systematic results in this direction can be found, for instance, in Karatzas and Shreve (1991) and Jacod et al. (2000). Fortunately, martingale representations exist for most integrable benchmarked contingent claims in Markovian market models and for most continuous market models, as will be demonstrated in the next section. A representation of a benchmarked contingent claim, which separates its self-financing hedgeable part from its orthogonal monitoring part, is crucial for hedging. We introduce the following notion:

Definition 6.1. We call a benchmarked contingent claim $\hat{H}_{T} \in \mathcal{L}^{1}\left(\mathcal{F}_{T}\right)$ regular if it has for all $t \in[0, T]$ a representation of the following form:

$$
\hat{H}_{T}=E\left(\hat{H}_{T} \mid \mathcal{F}_{t}\right)+\int_{t}^{T} \boldsymbol{\vartheta}_{\hat{H}_{T}}^{\top}(s) d \hat{\mathbf{S}}_{s}+\eta_{\hat{H}_{T}}(T)-\eta_{\hat{H}_{T}}(t)
$$

P-a.s, involving some predictable vector process $\boldsymbol{\vartheta}_{\hat{H}_{T}}=\left\{\boldsymbol{\vartheta}_{\hat{H}_{T}}(t)=\right.$ $\left.\left(\vartheta_{\hat{H}_{T}}^{1}(t), \ldots, \vartheta_{\hat{H}_{T}}^{d}(t)\right)^{\top}, t \in[0, T]\right\}$ satisfying $(2.6)$, and some local martingale 
$\eta_{\hat{H}_{T}}=\left\{\eta_{\hat{H}_{T}}(t), t \in[0, T]\right\}$ with $\eta_{\hat{H}_{T}}(0)=0$. Furthermore, the product process $\mathbf{Z}_{\hat{H}_{T}}=\left\{\mathbf{Z}_{\hat{H}_{T}}(t)=\eta_{\hat{H}_{T}}(t) \hat{\mathbf{S}}_{t}, t \in[0, T]\right\}$ forms a vector local martingale.

By combining Definition 5.3, Corollary 5.4 and Definition 6.1, benchmarked risk minimization allows us to obtain in a straightforward manner the following result:

Corollary 6.2. For a regular benchmarked contingent claim $\hat{H}_{T} \in \mathcal{L}^{1}\left(\mathcal{F}_{T}\right)$ with representation (6.1) there exists a BRM strategy $\boldsymbol{v}=\left\{\boldsymbol{v}_{t}=\right.$ $\left.\left(\eta_{\hat{H}_{T}}(t), \vartheta_{\hat{H}_{T}}^{1}(t), \ldots, \vartheta_{\hat{H}_{T}}^{d}(t)\right)^{\top}, t \in[0, T]\right\} \in \mathcal{V}_{\hat{H}_{T}}$ with corresponding benchmarked

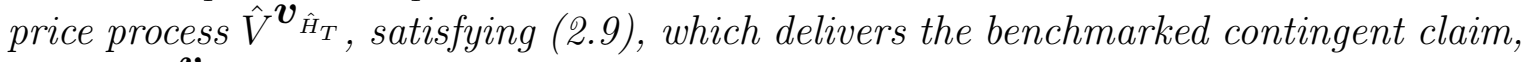
that is, $\hat{V}_{T}^{\boldsymbol{v}_{T}}=\hat{H}_{T}$ P-a.s. The benchmarked price at time $t \in[0, T]$ is determined by the real world pricing formula

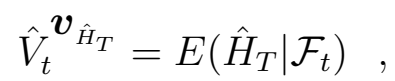

yielding within the set $\mathcal{V}_{\hat{H}_{T}}$ of admissible strategies the minimal possible price process. The resulting benchmarked $P \& L$ at time $t \in[0, T]$ is given by

$$
\hat{C}_{t}^{\boldsymbol{v}_{\hat{H}_{T}}}=\eta_{\hat{H}_{T}}(t)
$$

What remains is to identify for a given market model and given regular benchmarked contingent claim the respective representation of the form (6.1). To establish such representation, as a first step one can calculate the conditional expectation (6.2), either by explicit calculations or via some numerical methods. In a second step, one can identify the holdings $\boldsymbol{\vartheta}_{\hat{H}_{T}}$ in the self-financing part of the calculated benchmarked price process $\hat{V}^{\boldsymbol{v}_{\hat{H}_{T}}}$. The vector $\boldsymbol{\vartheta}_{\hat{H}_{T}}(t)$, characterizing the units to be held in the primary security accounts, follows by making the local martingale $\eta_{\hat{H}_{T}}(t)=\hat{V}_{t} \boldsymbol{v}_{\hat{H}_{T}}-\boldsymbol{\vartheta}_{\hat{H}_{T}}^{\top}(t) \hat{\mathbf{S}}_{t}$ orthogonal to the benchmarked primary security accounts. This means, the product $\eta_{\hat{H}_{T}}(t) \hat{\mathbf{S}}_{t}$ needs to form a driftless vector process. Note that due to the possible presence of redundant primary security accounts $\boldsymbol{\vartheta}_{\hat{H}_{T}}$ may not be unique. The final third step calculates then the units of the benchmark to be accumulated in the benchmarked P\&L.

\section{$7 \quad$ Hedging Regular Claims}

We emphasize BRM strategies do not request square integrability of benchmarked quantities. The benchmarked self-financing part and also the benchmarked P\&L do only need to form local martingales. Therefore, due to the avoidance of the request on the existence of an equivalent risk neutral probability measure, the proposed concept has wide applicability. As we will show in Section 9, it generalises important pricing rules and allows us to go far beyond the classical no-arbitrage modeling world. In particular, market models with jumps can be covered that may have infinite jump 
activity and random jump sizes. Interesting properties of BRM strategies emerge when studying particular types of models. It is impossible to present and discuss in this paper interesting results that emerge for models with jumps. A forthcoming paper will focus on such results and also on models where an equivalent risk neutral probability measure does not exist. In the remainder of the current paper we focus on BRM strategies for continuous models.

Without loss of generality, consider a benchmarked contingent claim $\hat{H}_{T}$ with fixed maturity, where all its uncertainty is modeled by the continuous local martingales $W^{1}, W^{2}, \ldots, W^{d}$. These local martingales are assumed to be orthogonal to each other in the sense that their pairwise products form local martingales. Furthermore, each benchmarked primary security account value $\hat{S}_{t}^{j}, j \in\{1, \ldots, d\}$, satisfies a stochastic differential equation of the form

$$
d \hat{S}_{t}^{j}=-\hat{S}_{t}^{j} \sum_{k=1}^{d-1} \theta_{t}^{j, k} d W_{t}^{k}
$$

for $t \geq 0$ with $\hat{S}_{0}^{j}>0$. Here $\theta^{j, k}=\left\{\theta_{t}^{j, k}, t \in[0, T]\right\}$ forms for each $j \in\{1, \ldots, d\}$ and $k \in\{1, \ldots, d-1\}$ a predictable process such that the Itô integrals corresponding to (7.1) exist. Note that the local martingale $W^{d}$ does not appear as uncertainty of the benchmarked primary security accounts. However, we allow it to model uncertainty of the benchmarked contingent claim $\hat{H}_{T}$. This means that the claim $\hat{H}_{T}$ will not be fully hedgeable.

To identify below the corresponding BRM strategy, denote by $\boldsymbol{\Phi}_{t}=\left[\Phi_{t}^{i, k}\right]_{i, k=1}^{d}$ the $d \times d$ matrix with elements

$$
\Phi_{t}^{i, k}=\left\{\begin{array}{cl}
\theta_{t}^{i, k} & \text { for } k \in\{1, \ldots, d-1\} \\
1 & \text { for } k=d
\end{array}\right.
$$

for $i \in\{1, \ldots, d\}$ and $t \in[0, T]$.

Proposition 7.1. In the setting of this section assume $\boldsymbol{\Phi}_{t}$ to be invertible for Lebesguealmost every $t \in[0, T]$. Furthermore, at time $t \in[0, T]$ the conditional expectation $\hat{V}_{t}$ of the benchmarked contingent claim is assumed to have a representation of the form

$$
\hat{V}_{t}=\hat{V}_{0}+\sum_{k=1}^{d-1} \int_{0}^{t} x_{s}^{k} d W_{s}^{k}+\int_{0}^{t} x_{s}^{d} d W_{s}^{d},
$$

where $x^{1}, \ldots, x^{d}$ are predictable processes. Then $\hat{H}_{T}$ is a regular benchmarked contingent claim with $E\left(\hat{H}_{T} \mid \mathcal{F}_{t}\right)=\hat{V}_{t}$ for all $t \in[0, T]$, and the corresponding BRM strategy is given by

$$
\boldsymbol{\vartheta}_{\hat{H}_{T}}(t)=\operatorname{diag}\left(\hat{\mathbf{S}}_{t}\right)^{-1}\left(\boldsymbol{\Phi}_{t}^{\top}\right)^{-1} \boldsymbol{\xi}_{t}
$$

with

$$
\boldsymbol{\xi}_{t}=\left(-x_{t}^{1}, \ldots,-x_{t}^{d-1}, \hat{V}_{t}-\eta_{\hat{H}_{T}}(t)\right)^{\top}
$$


and

$$
\eta_{\hat{H}_{T}}(t)=\int_{0}^{t} x_{s}^{d} d W_{s}^{d}
$$

The proof of this result is given in Appendix C. For instance, in a multi-factor Markovian diffusion model, which models the benchmarked contingent claim $\hat{H}_{T}$ and the benchmarked primary security accounts, one obtains in a straightforward manner a representation of the form (7.3) via the Feynman-Kac formula and by using the Kolmogorov backward equation for $\hat{V}_{t}$ as a function of the Markovian state variables. The application of the Itô formula to the pricing function provides directly the representation 7.3 .

The question arises, how does the above pricing and hedging relate to the wellknown hedging under the risk neutral approach? Note that when $W^{1} \ldots, W^{d}$ are independent standard Brownian motions, then $\theta_{t}^{i, k}$ becomes the market price of risk at time $t$ with respect to the $k$ th Brownian motion for the denomination of the securities in units of the $i$ th primary security account.

By the Itô formula it follows from (7.1) that the $j$ th primary security account, when denominated in units of the $i$ th primary security account, denoted by $\bar{S}_{t}^{i, j}=\frac{\hat{S}_{t}^{j}}{\hat{S}_{t}^{i}}$, satisfies the SDE

$$
d \bar{S}_{t}^{i, j}=\bar{S}_{t}^{i, j} \sum_{k=1}^{d-1}\left(\theta_{t}^{i, k}-\theta_{t}^{j, k}\right)\left(\theta_{t}^{i, k} d t+d W_{t}^{k}\right)
$$

for $t \in[0, T]$ and $i, j \in\{1, \ldots, d\}$. This shows that the volatility $b_{t}^{i, j, k}$ with respect to the $k$ th Brownian motion for the $j$ th primary security account, when denominated in units of the $i$ th primary security account, has the form

$$
b_{t}^{i, j, k}=\left(\theta_{t}^{i, k}-\theta_{t}^{j, k}\right)
$$

This also means, when we select, without loss of generality, the $d$ th primary security account as domestic savings account, then the volatility matrix $\mathbf{b}_{t}^{d}$ for the $d$ th security denomination has the form

$$
\mathbf{b}_{t}^{d}=\left[b_{t}^{d, j, k}\right]_{j, k=1}^{d-1, d-1}
$$

for $t \in[0, T]$.

It is well-known how one can hedge claims in the $d$ th security denomination. In this setting, the key assumption is that the volatility matrix $\mathbf{b}_{t}^{d}$ is an invertible matrix, see Karatzas and Shreve (1998). The following result shows that the matrix $\mathbf{b}_{t}^{d}$ is indeed invertible under our assumptions.

Proposition 7.2. For $t \in[0, \infty)$ the matrix $\boldsymbol{\Phi}_{t}$ is invertible if and only if $\mathbf{b}_{t}^{d}$ is invertible.

We provide the proof for this result in Appendix D. 


\section{A Quadratic Criterion}

The concept of benchmarked risk minimization avoids restrictive assumptions, which makes it widely applicable. However, its assumptions may appear rather abstract to some readers. Therefore, we show now that it can be interpreted, under appropriate assumptions, as the minimization of a "distance", which is the expected square of the benchmarked P\&L. The orthogonality of the benchmarked P\&L corresponds then to the minimization of its "distance" to benchmarked traded wealth.

To illustrate the link of BRM strategies to the indicated quadratic criterion, let us consider a regular benchmarked contingent claim $\hat{H}_{T}$, with $T \in(0, \infty)$ fixed, and representation 6.1). We assume in 6.1 that the terms $\int_{0}^{t} \boldsymbol{\vartheta}_{\hat{H}_{T}}^{\top}(s) d \hat{\mathbf{S}}_{s}$ and $\eta_{\hat{H}_{T}}(t)$ form independent, square integrable martingales. Additionally, assume that also $\hat{S}_{t}^{1}, \ldots, \hat{S}_{t}^{d}$ and $\eta_{\hat{H}_{T}}$ are mutually independent, square integrable martingales. The latter property guarantees that $\eta_{\hat{H}_{T}}$ is orthogonal to benchmarked traded primary security accounts, in the sense of Definition 5.1 .

Assume now that $\hat{H}_{T}$ is square integrable so that a square integrable martingale is formed by the conditional expectation $E\left(\hat{H}_{T} \mid \mathcal{F}_{t}\right)$. The second moment of the benchmarked P\&L represents the above mentioned "distance". Obviously, it can be interpreted as a measure for the risk of the hedge. This "distance" would be zero if the claim could be perfectly replicated. Now, let us minimize the above mentioned "distance", that is, we minimize

$$
E\left(\left(\hat{C}_{T}^{\delta}\right)^{2}\right)=E\left(\left(\hat{H}_{T}-\int_{0}^{T} \boldsymbol{\delta}_{s}^{\top} d \hat{\mathbf{S}}_{s}-\hat{S}_{0}^{\delta}\right)^{2}\right)
$$

by employing self-financing strategies $\boldsymbol{\delta}=\left\{\boldsymbol{\delta}_{t}=\left(\delta_{t}^{1}, \ldots, \delta_{t}^{d}\right)^{\top}, t \in[0, T]\right\}$, where $\int_{0}^{T} \boldsymbol{\delta}_{s}^{\top} d \hat{\mathbf{S}}_{s}$ is a square integrable martingale, independent of $\eta_{\hat{H}_{T}}$.

By exploiting the martingale representation (6.1), the orthogonality of $\eta_{\hat{H}_{T}}$ to benchmarked traded wealth and the assumed independence and square integrability properties, it follows that

$$
\begin{aligned}
& E\left(\left(\hat{C}_{T}^{\delta}\right)^{2}\right)=E\left(\left(E\left(\hat{H}_{T} \mid \mathcal{F}_{0}\right)-\hat{S}_{0}^{\delta}+\int_{0}^{T}\left(\boldsymbol{\vartheta}_{\hat{H}_{T}}^{\top}(s)-\boldsymbol{\delta}_{s}^{\top}\right) d \hat{\mathbf{S}}_{s}+\eta_{\hat{H}_{T}}(T)\right)^{2}\right) \\
& =E\left(E\left(\hat{H}_{T} \mid \mathcal{F}_{0}\right)-\hat{S}_{0}^{\delta}\right)^{2}+E\left(\int_{0}^{T}\left(\boldsymbol{\vartheta}_{\hat{H}_{T}}^{\top}(s)-\boldsymbol{\delta}_{s}^{\top}\right)^{2} d[\hat{\mathbf{S}}]_{s}\right)+E\left(\left(\eta_{\hat{H}_{T}}(T)\right)^{2}\right) .
\end{aligned}
$$

When minimizing the right hand side of the above equation it becomes obvious that the minimum can only be obtained when setting the benchmarked initial price to $\hat{S}_{0}^{\delta}=$ $E\left(\hat{H}_{T} \mid \mathcal{F}_{0}\right)$, which represents the price $\hat{V}_{0} \hat{\boldsymbol{H}}_{T}$ obtained by the real world pricing formula (6.2). Furthermore, taking the minimum requires choosing the second summand such that $\boldsymbol{\delta}_{t}=\boldsymbol{\vartheta}_{\hat{H}_{T}}(t)$ for all $t \in[0, T]$. We can not reduce the third summand in the above equation. Therefore, the minimal "distance" equals the minimal second moment for the benchmarked P\&L, which becomes $E\left(\left(\eta_{\hat{H}_{T}}(T)\right)^{2}\right)$. 
To extend this discussion, one could pool an increasing number of independent benchmarked P\&Ls of the above type in a trading book. This setup would satisfy the assumptions of Proposition 4.3, and the resulting total benchmarked P\&L would vanish almost surely. In this manner, a well-diversified institution can, in principle, remove asymptotically the nonhedgeable uncertainty from its trading book. The concept of benchmarked risk minimization identifies the hedging strategies yielding minimal fluctuations of benchmarked P\&Ls and, thus, allows one to perform systematically diversification in an optimal manner.

\section{$9 \quad$ Real World and Risk Neutral Pricing}

Let us interpret $\hat{S}^{1}$ as the benchmarked savings account process of the domestic currency. Obviously, it is a local martingale but may not be a true martingale. We can state the following result:

Proposition 9.1. For a benchmarked contingent claim $\hat{H}_{T}=\frac{H_{T}}{S_{T}^{1, \delta *}}$, with $S_{T}^{1, \delta *} d e-$ noting the value of the numéraire portfolio (NP) denominated in domestic currency at maturity $T$, the real world price coincides with the risk neutral price if the benchmarked savings account $\hat{S}^{1}$ is a true martingale, and the Radon-Nikodym derivative $\Lambda_{t}=\left.\frac{d Q}{d P}\right|_{\mathcal{F}_{t}}$ for the putative risk neutral measure $Q$ equals the normalized benchmarked savings account $\Lambda_{t}=\frac{\hat{S}_{t}^{1}}{\hat{S}_{0}^{1}}$, for $t \in[0, T]$.

Proof. The real world pricing formula $(6.2)$ can be rewritten for the discounted price process $\bar{S}_{t}^{\delta_{\hat{H}_{T}}}=\frac{\hat{S}_{t}^{\delta_{\hat{H}}}}{\hat{S}_{t}^{1}}$ by using Bayes's rule in the form

$\bar{S}_{t}^{\delta_{\hat{H}_{T}}}=\frac{S_{t}^{1, \delta *}}{S_{t}^{1,1}} E\left(\frac{H_{T}}{S_{T}^{1, \delta *}} \mid \mathcal{F}_{t}\right)=E\left(\frac{\hat{S}_{T}^{1}}{\hat{S}_{t}^{1}} \frac{H_{T}}{S_{T}^{1,1}} \mid \mathcal{F}_{t}\right)=E\left(\frac{\Lambda_{T}}{\Lambda_{t}} \frac{H_{T}}{S_{T}^{1,1}} \mid \mathcal{F}_{t}\right)=E^{Q}\left(\frac{H_{T}}{S_{T}^{1,1}} \mid \mathcal{F}_{t}\right)$.

Here $S_{t}^{1,1}=\hat{S}_{t}^{1} S_{t}^{1, \delta *}, t \in[0, T]$ denotes the savings account denominated in units of the domestic currency. The last equality on the right hand side of the above equation follows by the Bayes rule and provides the well-known risk neutral pricing formula, where $E^{Q}$ denotes expectation under $Q$.

The Radon-Nikodym derivative for the minimal equivalent martingale measure $Q$, as defined in Schweizer (1995), is characterized by the normalized benchmarked savings account $\Lambda_{t}=\left.\frac{d Q}{d P}\right|_{\mathcal{F}_{t}}=\frac{\hat{S}_{t}^{1}}{\hat{S}_{0}^{1}}$. Therefore, under the existence of the minimal equivalent martingale measure, classical risk minimization in the sense of Föllmer-SondermannSchweizer yields the same price as benchmarked risk minimization, which is a satisfying result. This does not mean that one obtains also the same hedging strategy, as we will see in the next section.

We remark that, it has been shown in Platen and Heath (2006) Section 9.2, that for $H_{T}$ independent of $S_{T}^{1, \delta *}$, the real world pricing formula yields the actuarial pricing 
formula, which has been widely used by actuaries without formal proof. The current paper provides a foundation for actuarial pricing via benchmarked risk minimization in incomplete markets and for nonhedgeable claims in a wide range of market models. Finally, we remark that it has been shown in Section 11.4 of Platen and Heath $(2006)$ that also some form of utility indifference pricing is equivalent to real world pricing.

\section{Differences between Classical and BRM Hedging}

We have seen in the previous section that when a minimal equivalent martingale measure exists, then real world pricing yields the same prices as classical risk minimization. However, this does not mean that after a hedge has been initiated using that price that both approaches yield the same hedging strategy. We demonstrate below that the hedging strategy for not fully hedgeable claims is different under the two approaches. The reason for this difference is the fact that the BRM strategy generates the benchmarked P\&L in such a way that it becomes orthogonal to the benchmarked primary security accounts under the real world probability measure. This is, in general, different to requesting that the discounted profit and loss is orthogonal to the discounted primary security accounts under the minimal equivalent martingale measure.

Proposition 10.1. BRM hedging of not fully replicable contingent claims is, in general, different to hedging under classical risk minimization using the minimal equivalent martingale measure.

Proof. As proof for the above statement we provide an illustrative example.

Consider in the setting of Section 7 with $d=2$ a random payout $H_{T}$, denominated in units of the domestic savings account $S_{t}^{1,1}$, such that $\hat{H}_{T}=\frac{H_{T} S_{T}^{1,1}}{S_{T}^{1, \delta *}}=H_{T} \hat{S}_{T}^{1}$ is a regular benchmarked contingent claim. More precisely, we assume that this discounted payout $H_{T}$ has the representation

$$
H_{T}=\exp \left\{-\frac{T}{2}+W_{T}^{2}\right\}
$$

where $W^{2}$ denotes the non-hedgeable Brownian motion.

On the other hand, we have in our example as primary security accounts the discounted savings account $S_{t}^{1,1}=1$ and the risky security $S_{T}^{1,2}$. The latter is in our example also the discounted NP, where we assume

$$
S_{t}^{1,2}=\exp \left\{\frac{t}{2}-W_{t}^{1}\right\}
$$

for $t \in[0, T]$, where $W^{1}$ denotes the hedgeable Brownian motion. The benchmarked savings account equals then $\hat{S}_{t}^{1}=\frac{1}{S_{t}^{1,2}}$, and the benchmarked NP is trivially $\hat{S}_{t}^{2}=1$ for 
$t \in[0, T]$. Obviously, from the perspective of classical risk minimization, the claim $H_{T}$ is not hedgeable in this market. According to Schweizer (2000), the minimal equivalent martingale measure $Q$ has the Radon-Nikodym derivative $\Lambda_{t}=\hat{S}_{t}^{1}$. Therefore, the discounted initial price for the claim amounts to

$$
V_{0}=E_{Q}\left(H_{T}\right)=E\left(\Lambda_{T} H_{T}\right)=E\left(\Lambda_{T}\right) E\left(H_{T}\right)=E\left(H_{T}\right)=1 .
$$

The hedging strategy under classical risk minimization would purchase at the initial time one unit of the savings account and would keep it until maturity.

The BRM strategy would, by Proposition 7.1, obtain the same initial price

$$
V_{0}=\hat{V}_{0}^{\hat{H}_{T}} S_{0}^{1, \delta *}=S_{0}^{1,2} \hat{V}_{0}^{\hat{H}_{T}}=S_{0}^{1,2} E\left(\frac{H_{T}}{S_{T}^{1, \delta *}}\right)=S_{0}^{1,2} E\left(H_{T}\right) E\left(\hat{S}_{T}^{1}\right)=E\left(H_{T}\right)=1 .
$$

Note that the conditional expectation of the benchmarked contingent claim equals

$$
\hat{V}_{t}=\hat{H}_{t}=E\left(\hat{H}_{T} \mid \mathcal{F}_{t}\right)=E\left(H_{T} \mid \mathcal{F}_{t}\right) E\left(\hat{S}_{T}^{1} \mid \mathcal{F}_{t}\right)=H_{t} \hat{S}_{t}^{1},
$$

with exponential martingales

$$
H_{t}=E\left(H_{T} \mid \mathcal{F}_{t}\right)=\exp \left\{-\frac{t}{2}+W_{t}^{2}\right\}
$$

and

$$
\hat{S}_{t}^{1}=\exp \left\{-\frac{t}{2}+W_{t}^{1}\right\}
$$

for $t \in[0, T]$. By the Itô formula, we obtain with (10.1) and 10.2 the martingale representation

$$
\begin{aligned}
\hat{V}_{t} & =H_{0} \hat{S}_{0}^{1}+\int_{0}^{t} H_{s} d \hat{S}_{s}^{1}+\int_{0}^{t} \hat{S}_{s}^{1} d H_{s} \\
& =H_{0} \hat{S}_{0}^{1}+\int_{0}^{t} H_{s} \hat{S}_{s}^{1} d W_{s}^{1}+\int_{0}^{t} \hat{S}_{s}^{1} H_{s} d W_{s}^{2}
\end{aligned}
$$

for $t \in[0, T]$. Consequently, we have in Proposition $7.1 x_{t}^{1}=H_{t} \hat{S}_{t}^{1}$ for $t \in[0, T]$. The $2 \times 2$ matrix $\boldsymbol{\Phi}_{t}$ has by $(7.2)$ and $(10.2)$ the form

$$
\boldsymbol{\Phi}_{t}=\left(\begin{array}{rr}
-1 & 1 \\
0 & 1
\end{array}\right)
$$

which equals its inverse $\boldsymbol{\Phi}_{t}^{-1}=\boldsymbol{\Phi}_{t}$ for $t \in[0, T]$. Furthermore, by (7.6), 10.3 and 7.5 we have $\eta_{\hat{H}_{T}}(t)=\int_{0}^{t} \hat{S}_{s}^{1} H_{s} d W_{s}^{2}$ and $\left(\boldsymbol{\xi}_{t}\right)^{\top}=\left(-H_{t} \hat{S}_{t}^{1}, H_{t} \hat{S}_{t}^{1}-\eta_{\hat{H}_{T}}(t)\right)$. By 7.4), (10.4) and $\hat{S}_{t}^{2}=1$ we get

$$
\vartheta_{\hat{H}_{T}}^{1}(t)=H_{t}
$$


and

$$
\vartheta_{\hat{H}_{T}}^{2}(t)=-\eta_{\hat{H}_{T}}(t) .
$$

We observe that this hedging strategy is different to the classical risk minimizing one. According to 10.5 the number of units held in the savings account equals $H_{t}=E\left(H_{T} \mid \mathcal{F}_{t}\right)$, which is the best forecast for the payoff $H_{T}$. Under classical risk minimization one holds always $H_{0}$ units in the savings account and nothing in any other security. Under the minimal equivalent martingale measure we still have a martingale for $\eta_{\hat{H}_{T}}(t)\left(\hat{S}_{t}^{1}\right)^{-1}$, which is the discounted P\&L of the BRM strategy. However, when multiplied with the discounted NP $S_{t}^{1, \delta *}=\left(\hat{S}_{t}^{1}\right)^{-1}$, which is a traded security, the product does not form a local martingale under the minimal equivalent martingale measure. This means, we do not have the kind of orthogonality that classical risk minimization requests. Alternatively, one can say, the discounted profit and loss $H_{t}-H_{0}$ at time $t \in[0, T]$ of the Föllmer-Schweizer decomposition, when multiplied by the benchmarked savings account $\hat{S}_{t}^{1}$, does not yield the benchmarked P\&L of the representation (6.1) of the regular benchmarked contingent claim. The claim we considered has a representation of a regular benchmarked contingent claim, as shown in (10.3).

This proves Proposition 10.1 .

This example demonstrates that BRM strategies take evolving information about the nonhedgeable uncertainty into account by using its best forecast, whereas classical risk minimization ignores such information. This is a key feature of the proposed concept of benchmarked risk minimization.

To satisfy investors who would like to minimize the second moment of their discounted $\mathrm{P} \& \mathrm{~L}$ under the minimal equivalent martingale measure, one can directly generalize classical risk minimization under the benchmark approach. Since this generalization is beyond the scope of the current paper it will be described in forthcoming work.

\section{Conclusion}

This paper proposes the concept of benchmarked risk minimization for pricing and hedging of not fully replicable contingent claims in incomplete markets. Benchmarked risk minimization goes beyond classical risk minimization, originally developed by Föllmer, Sondermann and Schweizer. Under the proposed concept a wider range of contingent claims can be priced and hedged in a richer modeling world. It does not require an equivalent risk neutral probability measure or square integrability properties. The main assumption is extremely weak. It only requires that the numéraire portfolio exists. The numéraire portfolio is employed as numéraire and benchmark. The resulting price represents the minimal possible price. The benchmarked profit and loss is a local martingale and orthogonal to benchmarked traded wealth in the 
sense that the product of benchmarked profit and loss with each benchmarked primary security account forms a local martingale.

When using benchmarked risk minimization, the total benchmarked profit and loss of a large trading book with increasing number of sufficiently different contingent claims can, in principle, be removed asymptotically. In this sense, benchmarked risk minimization yields the minimal possible price and allows one to remove the nonhedgeable risk via diversification.

In the case when classical risk minimization can be applied, benchmarked risk minimization yields the same price process, however, it employs a hedging strategy which takes evolving information about the nonhedgeable uncertainty of the claim into account, whereas classical risk minimization ignores such information.

\section{Acknowledgement}

The authors like to thank the Referees and the Associate Editor for crucial suggestions. Furthermore, they express their thanks to Martin Schweizer and Hardy Hulley for valuable discussions on the topic of the paper.

\section{Appendix A:}

Lemma 11.1. Consider for $T \in[0, \infty)$ a benchmarked contingent claim $\hat{H}_{T} \in \mathcal{L}^{1}\left(\mathcal{F}_{T}\right)$ and a supermartingale $Y=\left\{Y_{t}, t \in[0, T]\right\}$ with $Y_{T}=\hat{H}_{T} P$-a.s., as well as a martingale $X=\left\{X_{t}, t \in[0, T]\right\}$ with $X_{T}=Y_{T}=\hat{H}_{T} P$-a.s. Then it follows for all $t \in[0, T]$ the inequality

$$
X_{t} \leq Y_{t}
$$

P-a.s.

Proof. Following Section 3 in Chapter II of Revuz and Yor (1999), we can prove the above result as follows:

By the supermartingale property of $Y$ we have

$$
Y_{t} \geq E\left(\hat{H}_{T} \mid \mathcal{F}_{t}\right)
$$

$P$-a.s. for all $t \in[0, T]$. On the other hand, by the martingale property of $X$ it follows

$$
X_{t}=E\left(\hat{H}_{T} \mid \mathcal{F}_{t}\right)
$$

for all $t \in[0, T]$. Consequently, one has by 11.1 and 11.2 for call $t \in[0, T]$ the inequality

$$
Y_{t} \geq X_{t}
$$

$P$-a.s., which proves the statement of the above lemma. 


\section{Appendix B:}

\section{Proof of Proposition 4.3 ;}

We apply Kolmogorov's Strong Law of Large Numbers, see Chapter IV Section 3 in Shiryaey (1984). By the martingale property of $\hat{C}^{\boldsymbol{v}_{l}}$, we have $E\left(\frac{\hat{C}_{t}}{\hat{V}_{0}} \boldsymbol{v}_{l}\right)=0$ for all $l \in\{1,2, \ldots\}$ and $t \in[0, T]$.

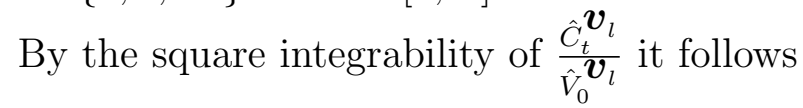

$$
\sum_{l=1}^{\infty} \frac{1}{l^{2}} E\left(\left(\frac{\hat{C}_{t}^{\boldsymbol{v}_{l}}}{\hat{V}_{0}^{\boldsymbol{v}_{l}}}\right)^{2}\right) \leq K_{t} \sum_{l=0}^{\infty} \frac{1}{l^{2}}<\infty
$$

$t \in[0, T]$. Thus, we obtain for $t \in[0, T]$ by the Strong Law of Large Numbers that

$$
\frac{\hat{R}_{m}(t)}{\hat{U}_{0}}=\frac{1}{m} \sum_{l=1}^{m} \frac{\hat{C}_{t}^{\boldsymbol{v}_{l}}}{\hat{V}_{0}^{\boldsymbol{v}_{l}}}
$$

converges $P$-almost surely to zero, which proves Proposition 4.3 .

\section{Appendix C:}

\section{Proof of Proposition 7.1:}

Denote by $\boldsymbol{\vartheta}_{t}$ the self-financing part of a BRM strategy $\boldsymbol{v} \in \hat{\mathcal{V}}_{\hat{H}_{T}}$. Accordingly, the self-financing part of the regular benchmarked claim $\hat{H}_{T}$ can be written as

$$
\begin{aligned}
\boldsymbol{\vartheta}_{t}^{\top} \hat{\mathbf{S}}_{t} & =\boldsymbol{\vartheta}_{t}^{\top} \operatorname{diag}\left(\hat{\mathbf{S}}_{t}\right) \mathbf{1} \\
& =\hat{V}_{t}-\int_{0}^{t} x_{s}^{d} d W_{s}^{d} .
\end{aligned}
$$

Furthermore, because $\boldsymbol{\vartheta}_{t}^{\top} \hat{\mathbf{S}}_{t}$ forms the self-financing part, one has by 7.1 the stochastic differential

$$
\begin{aligned}
\boldsymbol{\vartheta}_{t}^{\top} d \hat{\mathbf{S}}_{t} & =\boldsymbol{\vartheta}_{t}^{\top}\left(-\operatorname{diag}\left(\hat{\mathbf{S}}_{t}\right) \boldsymbol{\theta}_{t} d \mathbf{W}_{t}\right) \\
& =-\boldsymbol{\vartheta}_{t}^{\top} \operatorname{diag}\left(\hat{\mathbf{S}}_{t}\right) \boldsymbol{\theta}_{t} d \mathbf{W}_{t},
\end{aligned}
$$

where $\boldsymbol{\theta}_{t}=\left[\theta_{t}^{i, k}\right]_{1,1}^{d, d-1}$ is a $d \times d-1$ matrix, $\mathbf{W}_{t}=\left(W_{t}^{1}, \ldots, W_{t}^{d-1}\right)^{\top}$. By matching the self-financing part with the martingale representation (7.3), one has

$$
\boldsymbol{\vartheta}_{t}^{\top} \operatorname{diag}\left(\hat{\mathbf{S}}_{t}\right) \boldsymbol{\theta}_{t}=-\mathbf{x}_{t}^{\top}
$$

where $\mathbf{x}_{t}=\left(x_{t}^{1}, \ldots, x_{t}^{d-1}\right)^{\top}$ 
By 11.4 and $(11.5)$, one has with $\boldsymbol{\xi}_{t}=\left(-\mathbf{x}_{t}^{\top}, \hat{V}_{t}-\int_{0}^{t} x_{s}^{d} d W_{s}^{d}\right)^{\top}$ that

$$
\boldsymbol{\vartheta}_{t}^{\top} \operatorname{diag}\left(\hat{\mathbf{S}}_{t}\right) \boldsymbol{\Phi}_{t}=\boldsymbol{\xi}^{\top}
$$

Since $\boldsymbol{\Phi}_{t}$ is invertible, one obtains the relationship (7.4), which proves Proposition 7.1 .

\section{Appendix D:}

\section{Proof of Proposition 7.2 :}

Observe that the matrix $\boldsymbol{\Phi}_{t}$ has the form

$$
\boldsymbol{\Phi}_{t}=\left(\begin{array}{ccc|c}
\theta_{t}^{1,1} & \ldots & \theta_{t}^{1, d-1} & 1 \\
\vdots & \ddots & \vdots & \vdots \\
\theta_{t}^{d, 1} & \ldots & \theta_{t}^{d, d-1} & 1
\end{array}\right)
$$

We perform in the following operations that leave an invertible matrix invertible:

We are using the first until the $(d-1)$ th row from $\boldsymbol{\Phi}_{t}$, subtract from each the $d$ th row, and then take the negative elements in the resulting first until the $(d-1)$ th row. One obtains after these operations by (7.7) and (7.8) the matrix

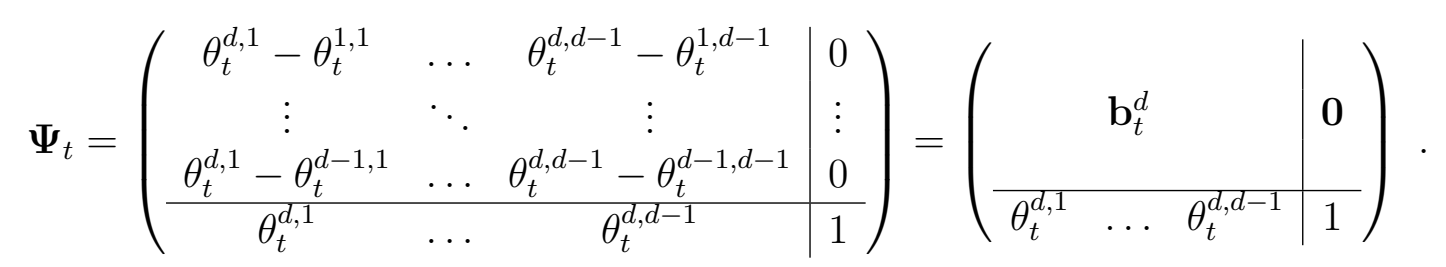

The matrix on the right hand side of the equation has in its upper left part the volatility matrix for the $d$ th denomination of the securities. Since we have in the matrix at the right hand side of the equation a "1" in the lower right hand corner, it is clear that the matrix $\mathbf{b}_{t}^{d}$ has full rank if and only if the matrix $\boldsymbol{\Psi}_{t}$ has full rank. Thus, it follows that $\boldsymbol{\Phi}_{t}$ has full rank if and only if $\mathbf{b}_{t}^{d}$ has full rank. Accordingly, the invertibility of $\boldsymbol{\Phi}_{t}$ is given if and only if $\mathbf{b}_{t}^{d}$ is invertible.

\section{References}

D. Becherer. The numéraire portfolio for unbounded semimartingales. Finance and Stochastics, 5:327-341, 2001.

F. Biagini. Evaluating hybrid products: the interplay between financial and insurance markets. Preprint, LMU Munich, 2011.

F. Biagini, A. Cretarola, and E. Platen. Local risk-minimization under the benchmark approach. Preprint, LMU Munich, 2011. 
M. M. Christensen and K. Larsen. No arbitrage and the growth optimal portfolio. Stochastic Analysis and Applications, 25(1):255-280, 2007.

M. H. A. Davis and S. Lleo. Fractional Kelly strategies for benchmarked asset management. In Mclean, Thorp \& Ziemba (Eds.) The Kelly Capital Growth Investment Criterion, Singapore: World Scientific, pages 385 - 407, 2011.

F. Delbaen and W. Schachermayer. Arbitrage possibilities in Bessel processes and their relations to local martingales. Probability Theory and Related Fields, 102:357-366, 1995.

D. Fernholz and I. Karatzas. Probabilistic aspects of arbitrage. In: Chiarella, C. \& Novikov, A. (Eds.) Contemporary Quantitative Finance, Springer Verlag, pages 1 19, 2010.

R. Fernholz. Stochastic Portfolio Theory. Springer-Verlag, New York., 2002.

R. Fernholz and I. Karatzas. Stochastic portfolio theory: An overview. Bensoussan, A. \& Zhang, Q. (eds): Mathematical Modeling and Numerical Methods in Finance, Handbook of Numerical Analysis, North-Holland, Oxford, XV:89 - 167, 2009.

R. Fernholz, I. Karatzas, and C. Kardaras. Diversity and relative arbitrage in equity markets. Finance and Stochastics, no. 9, pp. 127, 2005.

H. Föllmer and M. Schweizer. Hedging by sequential regression: an introduction to the mathematics of option trading. ASTIN Bulletin, 18:147 - 160, 1989.

H. Föllmer and D. Sondermann. Hedging of contingent claims under incomplete information. M. H. A. Davis and R. J. Elliott (eds.), "Applied Stochastic Analysis", Stochastics Monographs, 5:389 - 414, 1986.

G. Galesso and W. J. Runggaldier. Pricing without equivalent martingale measures under complete and incomplete observation. Chiarella, C. \& Novikov, A. (eds.), Contemporary Quantitative Finance, Springer, Berlin - Heidelberg, pages 99 - 121, 2010 .

David Heath and Eckhard Platen. Consistent pricing and hedging for a modified constant elasticity of variance model. Quantitative Finance, 2(6):459-467, 2002.

S. L. Heston, M. Loewenstein, and G. A. Willard. Options and bubbles. Review of Financial Studies, 20(2):359-390, 2007.

J. Jacod, S. Meleard, and P. Protter. Explicit form and robustness of martingale representations. The Annals of Probability, 28(4):pp. 1747-1780, 2000.

R. A. Jarrow, P. Protter, and K. Shimbo. Asset price bubbles in incomplete markets. Mathematical Finance, 20(2):145-185, 2010. 
I. Karatzas and C. Kardaras. The numéraire portfolio in semimartingale financial models. Finance and Stochastics, 11:447-493, 2007.

I. Karatzas and S. E. Shreve. Brownian Motion and Stochastic Calculus. Springer, 1991.

I. Karatzas and S. E. Shreve. Methods of Mathematical Finance, volume 39. Appl. Math. Springer, 1998.

J. Kelly. A new interpretation of information rate. Information Theory, IRE Transactions on, 2(3):185 -189, September 1956.

M. Loewenstein and G. A. Willard. Local martingales, arbitrage, and viability free snacks and cheap thrills. Economic Theory, 16:135-161, 2000.

J. B. Long. The numéraire portfolio. Journal of Financial Economics, 26(1):29 - 69, 1990.

E. Platen. Arbitrage in continuous complete markets. Advances in Applied Probability, 3:540 - 558, 2002.

E. Platen. A benchmark approach to finance. Mathematical Finance, 16(1):131-151, 2006.

E. Platen and D. Heath. A Benchmark Approach to Quantitative Finance. Springer, 2006.

P. E. Protter. Stochastic Integration and Differential Equations. 3rd edn, Springer, New York, 2005.

D. Revuz and M. Yor. Continuous Martingales and Brownian Motion. 3rd edn, Springer, 1999.

M. Schweizer. Option hedging for semimartingales. Stochastic Processes and their Applications, 37(2):339 - 363, 1991.

M. Schweizer. On the minimal martingale measure and the Föllmer-Schweizer decomposition. Stochastic Analysis and Applications, 13(5):573-599, 1995.

M. Schweizer. A guided tour through quadratic hedging approaches. E. Jouini, J. Cvitanic, M. Musiela (eds.), "Option Pricing, Interest Rates and Risk Management", Cambridge University Press, pages 538-574, 2000.

A. N. Shiryaey. Probability. Springer, 1984. 